\title{
c-Src is in the effector pathway linking uPAR and podocyte injury
}

\author{
Jeffrey B. Kopp and Jurgen Heymann \\ Kidney Disease Section, National Institute of Diabetes and Digestive and Kidney Diseases (NIDDK), NIH, Bethesda, Maryland, USA.
}

\begin{abstract}
The role of urokinase-type plasminogen activator receptor (UPAR) in kidney physiology and pathology has attracted considerable attention. The protein uPAR has dual functions: as a key regulator of plasmin generation and a component of the innate immune system. In the current issue, Wei and colleagues describe a transgenic mouse expressing Plaur RNA in glomerular podocytes. The mice manifested podocyte injury, including c-Src phosphorylation, proteinuria, and focal segmental glomerulosclerosis (FSCS). Plaur-transgenic mice on a $\beta_{3}$ integrin-deficient background were protected from podocyte injury. Renal biopsies from subjects with FSCS, but not those with other glomerular diseases, manifested increased c-Src phosphorylation in podocytes. These findings suggest a novel injury mechanism in FSCS, with possible implications for new treatment strategies.
\end{abstract}

\section{Role of uPAR in kidney} pathophysiology

Since 1995, more than 300 articles have been published on the role of urokinase-type plasminogen activator receptor (UPAR) in kidney physiology or pathology. The human PLAUR gene, encoding UPAR, is located on chromosome 19 and is part of the Ly6/PLAUR family. PLAUR gene expression in podocytes and other cells is driven by the DNA-binding protein Krüppel-like factor 4 (KLF4). This is likely part of a larger epigenetic program, as epigenetic remodeling of podocyte DNA by KLF4 attenuates renal injury in murine adriamycin nephropathy (1). UPAR is a glycosylphosphatidylinositol-anchored (GPI-anchored) protein that is located on the cell surface and has two distinct sets of functions. First, operating as an enzyme, it activates the plasminogen activator, which converts inactive plasminogen to plasmin, a serine protease. Plasmin participates in diverse processes, including fibrinolysis, complement activation, and extracellular matrix remodeling. Second, UPAR interacts with other cell surface proteins, including integrins; growth factor receptors; and the formyl peptide receptor, which is activated by peptides released by injured tissues and microorganisms (2). In this second capacity, uPAR contributes to innate immunity.

The proteins of the Ly6/PLAUR family share the structural feature of having one or more characteristic Ly6-urokinase plasminogen (LU) domains composed of 60-90 amino acids, each having a beta barrel core, stabilized by 4 disulfide bonds, and each having 3 extended loops. The PLAUR family includes a number of snake venoms, which evolved to bind particular vertebrate receptors, including nicotinic and muscarinic acetylcholine receptors, $\alpha$ - and $\beta$-adrenergic receptors, and others (3). Most LU protein family members have 7 exons, and some transcripts manifest alternate splicing. PLAUR RNA transcripts

Related Article: p. 1946

Conflict of interest: The authors have declared that no conflict of interest exists.

are most highly expressed in bone marrow, with lower levels of expression in gallbladder, urinary bladder, kidney, appendix, and placenta.

\section{Clinical and laboratory studies linking serum uPAR to podocyte injury}

Interest in UPAR was greatly heightened within nephrology with the publication in 2011 of an article by Wei, Reiser, and colleagues showing that levels of serum soluble UPAR (suPAR) were higher in certain patients with the multifaceted clinicalpathologic syndrome focal segmental glomerulosclerosis (FSGS), compared with other kidney diseases (4). The authors suggested that suPAR might be responsible for at least some cases of recurrent FSGS following kidney transplantation. They showed evidence supporting a mechanism that involved suPAR binding to integrin $\beta_{3}$ on the podocyte plasma membrane (Figure 1). Bone marrow plasma cells have been identified as a major source of circulating suPAR (5).

Recently, a group from Singapore reported on 55 children presenting with steroid-dependent nephrotic syndrome, all of whom underwent kidney biopsy (6). They found that serum suPAR levels were significantly higher among those with FSGS compared with those with minimal change disease (MCD) $(P<0.02)$, whereas suPAR levels were similar in MCD and healthy subjects. suPAR, estimated glomerular filtration rate (eGFR), and $\mathrm{CD}^{+}$ memory $\mathrm{T}$ cells were each of some value in predicting the presence of FSGS, but the combination of these 3 variables performed best (AUC 0.82, 95\% CI 0.690.95). Weighted risk scores improved the model. The model was also tested in subjects with steroid-resistant nephrotic syndrome, including MCD; these data are difficult to interpret, as individuals with MCD who do not respond to steroids likely have another condition, likely unsampled FSGS. With regard to basic studies, Dryer and 


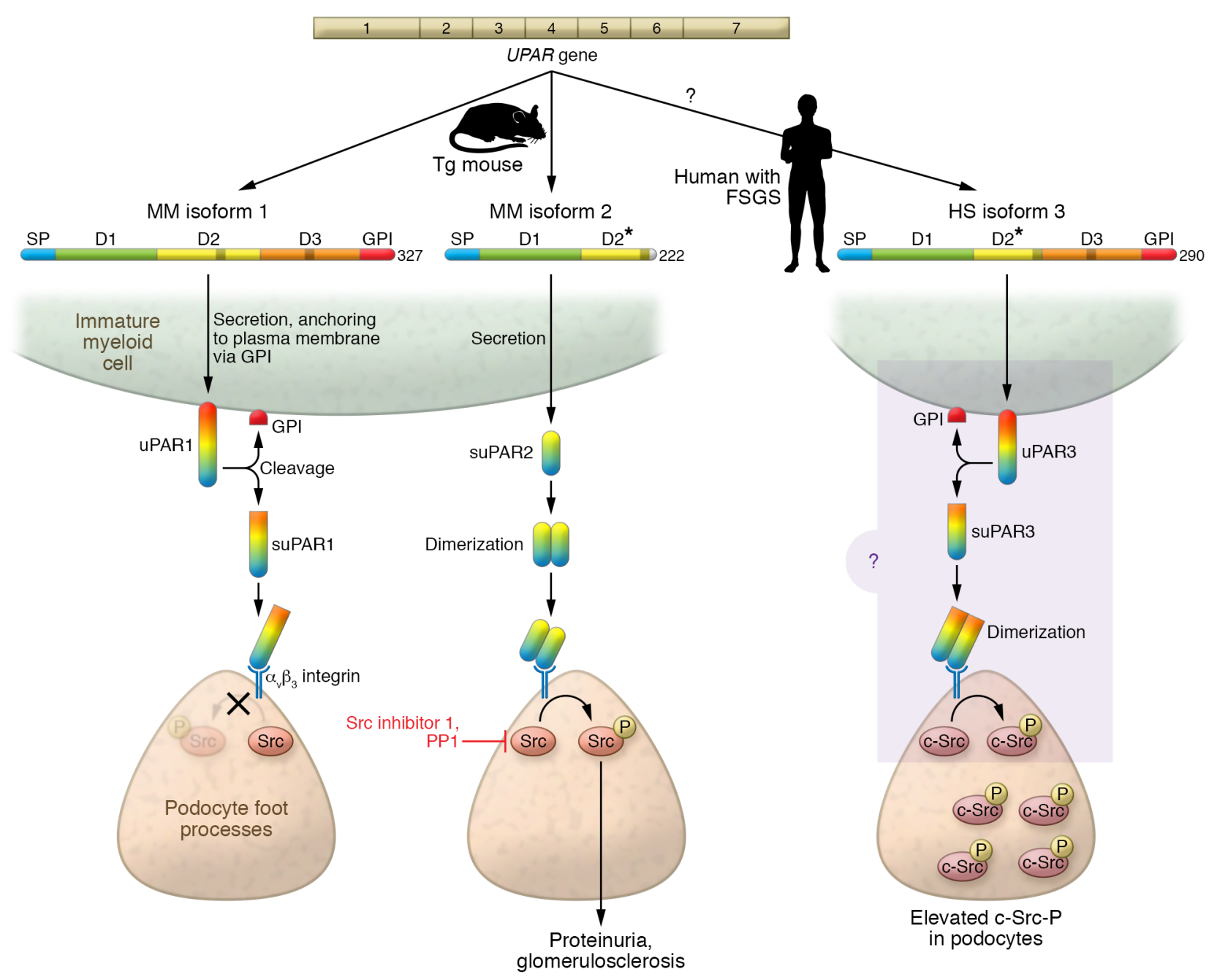

Figure 1. Mechanism of uPAR in podocyte injury. Mus musculus (MM) UPAR1 isoform 1 is secreted and anchored to the plasma membrane via the GPI anchor, and is then converted from a membrane-bound form (UPAR) into a soluble form (suPAR) by enzymatic removal of the GPI anchor. MM uPAR isoform 2 lacks a GPI anchor and hence is a suPAR; in contrast to MM isoform 1, it forms a soluble dimer. In mice transgenic for either MM isoform 1 or MM isoform 2 , suPAR binds $a_{4} \beta_{3}$ integrin located at the podocyte foot process plasma membrane. Binding of suPAR isoform 2, possibly as a dimer, generates phosphorylated c-Src, through outside-in signaling. By contrast, for isoform 1, no c-Src-P was detected. Transgenic mice bearing suPAR isoform 2 uniquely develop proteinuria and glomerulosclerosis. Targeting of c-Src with Src-specific inhibitors (Src inhibitor 1 or PP1) reduced proteinuria. Homo sapiens (HS) uPAR isoform 3 is the result of in-frame exon splicing leading to preservation of the GPI anchor and is predicted to form dimers, resembling MM isoform 2. Elevated levels of phosphorylated c-Src were found in human kidney biopsy samples, as with MM suPAR isoform 2 mice. This suggests that in human podocytes HS suPAR isoform 3 functions similarly to MM isoform 2, increasing phosphorylated c-Src, which drives the development of FSCS. D, domain. D2*, truncated D2.

colleagues recently demonstrated that suPAR causes activation of podocyte TRPC6 channels, resulting in calcium ion influx. This activity was dependent on calcium and was blocked by TEMPOL, suggesting a role for oxidative stress (7). Exposing the podocytes to $\mathrm{H}_{2} \mathrm{O}_{2}$ or suPAR increased tyrosine phosphorylation of many proteins, including cellular Src tyrosine kinase (c-Src). Further, the activation of TRPC6 channels by suPAR or oxidative stress was blocked by a c-Src inhibitor. The authors summarize an injury pathway thus: suPAR generates oxidative stress, which promotes signaling via c-Src phos- phorylation, leading to TRPC6 channel activation and increased cytosolic calcium levels. The combination of oxidative stress and calcium mobilization may compromise podocyte function and survival.

The hypothesis linking suPAR to podocyte injury and FSGS has great potential to advance our limited understanding of the mechanisms of primary FSGS. The role of suPAR in recurrent FSGS in particular and in podocyte injury in general has remained controversial, as summarized in two recent reviews $(8,9)$ and a letter to the editor $(10)$.

Some researchers have not been able to replicate these findings with regard to the role of suPAR in podocyte injury in mice, for reasons that remain unclear but may relate to different study populations or perhaps differences in assay methods and conditions (11). More work is required before the community can reach a consensus on the clinical role of suPAR testing in FSGS.

\section{SUPAR RNA isoform 2 overexpression induces proteinuria and FSCS}

In the current issue of the JCI, Wei and colleagues have extended their prior work in this area by characterizing the function of mouse Plaur RNA isoform 2 (12). 
The Plaur gene has 4 RNA transcript isoforms, encoding predicted proteins of 335, 281, 290, and 266 amino acid residues in length (protein isoforms 1-4). Prior experimental work by Reiser and colleagues has involved Plaur transcript 1, with 3 globular domains. In the current study, the group has generated transgenic mice expressing Plaur transcript 2 under the control of a mouse promoter for the adipocyte fatty acid binding protein (AP2). This PLAUR isoform only contains 1.5 globular domains (domain 1 and half of domain 2) and thus lacks the GPI anchor necessary for cell surface expression. The transgene was shown to be expressed in podocytes in vivo. Proteinuria and reduced serum albumin developed more rapidly in ap2/Plaur mice when a high-fat diet was used to stimulate ap2 transgene promoter activity. In the latter group, about one-quarter of the mice developed FSGS. When ap2/Plaur mice were crossed with $\beta_{3}$ integrin-knockout mice, the dual transgenic mice did not develop kidney disease, confirming an essential role for $\beta_{3}$ integrin in uPAR pathogenesis. As uPAR antibodies were not cross-reactive between humans and mice, it was not possible to determine whether UPAR levels in mice were comparable to those in patients with FSGS.

The investigators next examined the role of $\mathrm{c}$-Src in uPAR-induced podocyte injury. c-Src is a non-receptor tyrosine kinase and proto-oncogene that has diverse roles in development, cell survival and proliferation, angiogenesis, and tissue invasion. This was a logical direction to take, as for 2 decades c-Src has been known to be a downstream effector of PLAUR, including in aortic smooth muscle cells (13). Recently, $\gamma \delta \mathrm{T}$ cells have been shown to induce a CD28/c-Src pathway in podocytes (14).

Interestingly, Wei and colleagues found that UPAR isoform 2, but not UPAR isoform 1, activated c-Src. Treatment of proteinuric ap2/Plaur mice with an Src inhibitor reduced proteinuria by more than $50 \%$. These findings prompted the authors to examine phospho-c-Src expression in human glomerular disease by immunostaining; they found phosphoc-Src in podocytes in FSGS biopsies but not in MCD, membranoproliferative glomerulonephritis, or lupus nephritis biopsies. Finally, they showed that all 4 PLAUR RNA isoforms are present in human peripheral blood mononuclear cells. Taken together, these data add further support for a role for the signaling pathway suPAR $\rightarrow \alpha_{v} / \beta_{3}$ integrin $\rightarrow \mathrm{c}$-Src, in experimental glomerular disease and plausibly in human glomerular disease as well.

\section{Future directions}

What lies ahead for this line of investigation? It is interesting that among glomerular diseases studied, phospho-c-Src was restricted to FSGS. FSGS can be considered as 6 distinct syndromes whose pathogenesis involves many different mechanisms (15). Primary FSGS is likely associated with one or more plasma permeability factors, and suPAR may be one of these. It will be important to learn whether other forms of FSGS are associated with c-Src activation, the expression patterns of PLAUR RNA isoforms in clinical FSGS and other glomerular diseases, and the implications for therapeutic response and long-term outcomes.

\section{Acknowledgments}

The authors appreciate comments from Ben Afzali. This work was supported by the Intramural Research Program, NIDDK, NIH (Z1A DK043308).

Address correspondence to: Jeffrey B. Kopp, 10 Center Dr., 3N116, NIH, Bethesda, Maryland 20892-1268, USA. Phone: 301.594.3403; Email: jbkopp@nih.gov.
1. Hayashi K, et al. KLF4-dependent epigenetic remodeling modulates podocyte phenotypes and attenuates proteinuria. JClin Invest. 2014;124(6):2523-2537.

2. Montuori N, Ragno P. Multiple activities of a multifaceted receptor: roles of cleaved and soluble uPAR. Front Biosci (Landmark Ed). 2009;14:2494-2503.

3. Kini RM, Doley R. Structure, function and evolution of three-finger toxins: mini proteins with multiple targets. Toxicon. 2010;56(6):855-867.

4. Wei C, et al. Circulating urokinase receptor as a cause of focal segmental glomerulosclerosis. Nat Med. 2011;17(8):952-960.

5. Hahm E, et al. Bone marrow-derived immature myeloid cells are a main source of circulating suPAR contributing to proteinuric kidney disease. Nat Med. 2017;23(1):100-106.

6. Chan CY, et al. MeSsAGe risk score: tool for renal biopsy decision in steroid-dependent nephrotic syndrome. Pediatr Res. 2019;85(4):477-483.

7. Kim EY, Shotorbani PY, Dryer SE. TRPC6 inactivation does not affect loss of renal function in nephrotoxic serum glomerulonephritis in rats, but reduces severity of glomerular lesions. Biochem Biophys Rep. 2019;17:139-150.

8. Kronbichler A, Saleem MA, Meijers B, Shin JI. Soluble urokinase receptors in focal segmental glomerulosclerosis: a review on the scientific point of view. JImmunol Res. 2016;2016:2068691.

9. Saleem MA. What is the role of soluble urokinase-type plasminogen activator in renal disease? Nephron. 2018;139(4):334-341.

10. Mesnard L, Luque Y, Rondeau E. Experimental concerns regarding suPAR-related proteinuria. Nat Rev Nephrol. 2017;13(9):593.

11. Cathelin D, et al. Administration of recombinant soluble urokinase receptor per se is not sufficient to induce podocyte alterations and proteinuria in mice. J Am Soc Nephrol. 2014;25(8):1662-1668.

12. Wei $\mathrm{C}$, et al. uPAR isoform 2 forms a dimer and induces severe kidney disease in mice. JClin Invest. 2019;129(5):1946-1959.

13. Dumler I, et al. The Jak/Stat pathway and urokinase receptor signaling in human aortic vascular smooth muscle cells. J Biol Chem. 1998;273(1):315-321.

14. Chen W, Wu Y, Zhang G, Wang M, Yang H, Li Q. $\gamma \delta$ T cells exacerbate podocyte injury via the CD28/B7-1-Phosphor-SRC kinase pathway. Biomed Res Int. 2018;2018:5647120.

15. Rosenberg AZ, Kopp JB. Focal segmental glomerulosclerosis. Clin J Am Soc Nephrol. 2017;12(3):502-517. 